Full Length Article

\title{
Residual thrombin potential predicts cardiovascular death in acute coronary syndrome patients undergoing percutaneous coronary intervention
}

\author{
Monica Attanasio a,b,*, Rossella Marcucci a,b, Anna Maria Gori ${ }^{\mathrm{a}, \mathrm{b}, \mathrm{d}}$, Rita Paniccia ${ }^{\mathrm{a}, \mathrm{b}}$, Serafina Valente ${ }^{\mathrm{b}}$, \\ Daniela Balzi ${ }^{\mathrm{c}}$, Alessandro Barchielli ${ }^{\mathrm{c}}$, Nazario Carrabba ${ }^{\mathrm{b}}$, Renato Valenti ${ }^{\mathrm{b}}$, David Antoniucci ${ }^{\mathrm{b}}$, \\ Rosanna Abbate ${ }^{\mathrm{d}, \mathrm{e}, \mathrm{f}}$, Gian Franco Gensini ${ }^{\mathrm{d}, \mathrm{e}, \mathrm{f}}$ \\ a Department Experimental and Clinical Medicine, University of Florence, Italy \\ b Careggi University Hospital, Florence, Italy

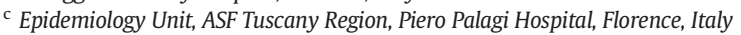 \\ d Don Gnocchi Foundation, Florence, Italy \\ e University of Florence, Italy \\ ${ }^{\mathrm{f}}$ CESMAV, Florence, Italy
}

\section{A R T I C L E I N F O}

\section{Article history:}

Received 27 June 2016

Received in revised form 5 September 2016

Accepted 18 September 2016

Available online 19 September 2016

\section{Keywords:}

Thrombin

Myocardial infarction

Coronary disease

Mortality

\begin{abstract}
A B S T R A C T
Introduction: Thrombin generation (TG) is a central step of the coagulation system involved in hemostatic and thrombotic roles. Scarce data evaluating in the acute phase the association between TG and the risk of cardiovascular death of acute coronary syndrome (ACS) patients are available, in the era of percutaneous coronary intervention $(\mathrm{PCI})$ and stenting with the use of dual antiplatelet treatment.

Materials and methods: We investigated TG in 292 ACS patients undergoing PCI with stent implantation on dual antiplatelet treatment. Venous samples were obtained 12-24 h after PCI. TG was assessed using the Calibrated Automated Thrombogram (CAT).

Results: At two years of follow-up, 57 out of 292 patients (19.5\%) died from cardiovascular causes. Higher values of endogenous thrombin potential (ETP) [1115.9 (705-1441.3) vs 940.2 (666.0-1253.1), $\mathrm{p}=0.049$ ], peak [176.1 (80.5-259.4) vs 107.3 (59.9-181.1), $\mathrm{p}=0.002$ ] and velocity index [61.75 (21.03-97.88) vs 25.64 (11.95-50.90), $\mathrm{p}<0.001$ ] were observed in relation to survival patients. At the multivariate model adjusted for the Global Registry of Acute Coronary Events risk score, the association between TG and cardiovascular death remained significant for ETP [OR (95\% CI): 2.58 (1.10-6.03), $\mathrm{p}=0.029]$, peak [OR (95\%CI): 3.27 (1.35-7.92), $\mathrm{p}=0.009$ ] and velocity index [OR $(95 \% \mathrm{CI}): 3.06(1.27-7.39), \mathrm{p}=0.013]$. This result was confirmed after adjustment for high on-treatment platelet reactivity [ETP: OR $(95 \% \mathrm{CI}) 2.35$ (1.11-5.00), p $=0.027$; peak: OR (95\% CI) 2.42 (1.13$5.15), \mathrm{p}=0.022$; velocity index: $\mathrm{OR}(95 \% \mathrm{CI}) 2.43$ (1.14-5.20), $\mathrm{p}=0.022$ ].

Conclusions: ACS patients with a residual TG after PCI and stent implantation have a significantly higher risk of long-term cardiovascular death. These results might be useful in improving risk stratification for ACS patients and support the need of a tailored antithrombotic therapy.
\end{abstract}

(c) 2016 Published by Elsevier Ltd.

\section{Introduction}

The most common underlying mechanism of acute coronary syndrome (ACS) is thrombus development on a damaged atherosclerotic plaque. Thrombin is a serine proteinase and is involved in hemostatic and thrombotic roles, from promotion and inhibition of clotting, to fibrinolysis, platelet activation, and inflammation. In the past, biochemical

\footnotetext{
* Corresponding author at: Department of Experimental and Clinical Medicine, University of Florence, Largo Brambilla 3, 50134 Florence, Italy.

E-mail address: monica.attanasio@unifi.it (M. Attanasio).
}

signs of coagulation activation in the acute phase of unstable angina or acute myocardial infarction (AMI) were demonstrated [1,2] and blood clotting activation markers were found to be independent risk factors for recurrence and long-term mortality [3-6]. In the last years the diffuse use of $\mathrm{PCl}$ and stenting stimulated several studies investigating primarily the role of platelet hyper-reactivity in determining clinical outcome $[7,8]$. On the other hand, scarce data are available on the possible role of blood clotting activation on the prognosis of ACS patients, in the era of PCI and stenting on the top of dual antiplatelet therapy. In 2011 in a small group of AMI patients the risk of recurrent ischemic cardiovascular complications was evaluated in relation to thrombin 
generation, but the low percentage of primary endpoints did not allow to obtain clear results [9]. In 2014 Schneider et al. investigated TG in a non-homogeneous cohort of CAD patients (unstable and stable coronary artery disease), performing the biohumoral evaluation in a large time interval from the index event, found an inverse association between TG and cardiovascular death [10]. Aim of our study was to evaluate the possible association between cardiovascular death and the entity of thrombin generation measured in the acute phase of ACS in patients undergone PCI and stenting. We measured thrombin generation (TG) by the Calibrated Automated Thrombogram (CAT) assay, which offers a global assessment of coagulation [11]. Residual platelet reactivity was also evaluated in relation to the risk of cardiovascular death.

\section{Methods}

The study population comprised a group of 421 patients discharged from the Coronary Unit of the Careggi University Hospital (Florence, Italy) from April 2008 to April 2010 with a diagnosis of acute coronary syndrome (ACS) and enrolled in the frame of the Florence Acute Myocardial Infarction-2 (AMI-Florence 2) registry [12]. Fifty patients were excluded as they interrupted dual antiplatelet therapy for major surgery (due to cardiovascular or liver disease or cancer) during the follow-up in order to avoid the inclusion of ischemic events possibly occurring for the therapy interruption. Sixty-six patients were lost at follow-up and 13 patients were excluded as they were on oral anticoagulant treatment. Therefore, the final study population included 292 ACS patients undergone PCI with stent implantation (155 ST-elevation myocardial infarction [STEMI]; 137 NSTEMI/Unstable Angina). In all these patients dual antiplatelet therapy was continued for 12 months. ACS was diagnosed according to criteria established by the European Society of Cardiology [13]. Unstable angina was defined as a history of new-onset, more frequent, more persistent or rest episode of chest pain, without typical changes of myocardial enzymes and with electrocardiogram evidence of myocardial ischemia (transient ST segment displacement $>0.1 \mathrm{mV}$ during chest pain).

All patients underwent coronary angiography performed by the Judkins' technique and percutaneous coronary intervention (PCI). Before $\mathrm{PCI}$, all patients received a loading dose of $500 \mathrm{mg}$ of acetylsalicylic acid (ASA) and $600 \mathrm{mg}$ of clopidogrel, followed by 100/325 mg of ASA daily and $75 \mathrm{mg}$ of clopidogrel daily. During PCI, standard activated clotting time (ACT)-guided doses of intravenous unfractioned heparin (UFH) were used (ACT: 250-300 s) after an $85 \mathrm{U} / \mathrm{kg}$ bolus of UFH.

Current smoking status was determined at the time of blood collection. The subjects were classified as having hypertension according to the guidelines of European Society of Hypertension/European Society of Cardiology [14] or if they reported taking antihypertensive medications, as verified by the physician. Diabetic subjects were defined in agreement with the American Diabetes Association [15] or on the basis of self-report data (if confirmed by medication or chart review). Dyslipidemia was defined according to the Third report of the National Cholesterol Education Program (NCEP-III) [16] or if they reported taking antidyslipidemic drugs, as verified by the physician. A positive family history was defined as the presence of at least one first-degree relative who had developed coronary artery disease (CAD) before the age of 55 years for men and 65 years for women. All subjects gave informed consent; the study complies with the Declaration of Helsinki and was approved by the local ethic committee.

Venous blood samples anticoagulated with $0.109 \mathrm{M}$ sodium citrate were taken between 12 and $24 \mathrm{~h}$ after PCI. Platelet poor plasma for the assessment of thrombin generation was obtained by double centrifugation at $2000 \times \mathrm{g}$ for $15 \mathrm{~min}$ at room temperature and stored at $80{ }^{\circ} \mathrm{C}$ until analysis.

As regard Calibrated Automated Thrombogram (CAT) assessment, we used the method described by Hemker and coworkers [17] and commercialized by Thrombinoscope BV, (Maastricht, the Netherlands). According to the manufacturer's instructions, measurements were conducted in $80 \mu \mathrm{l}$ of platelet poor plasma (PPP) triggered by $20 \mu \mathrm{l}$ PPP-reagent (tissue factor $5 \mathrm{pmol} / \mathrm{l}$ ) in 96-well microtiter plates. Measurements were calibrated against the fluorescence curve obtained in the same plasma mixed with $20 \mu \mathrm{l}$ Thrombin Calibrator (Thrombinoscope BV). Fluorogenic substrate (20 $\mu \mathrm{l}$ ) were added to sample and calibrator wells and the fluorescence intensity was detected in a Fluoroskan Ascent reader (Thermo Labsystems OY, Helsinki, Finland) with a 390/460 filter set for 60 min and the TG curves were calculated with Thrombinoscope software (Thrombinoscope BV). TG was expressed as endogenous thrombin potential (ETP: the area under the curve that represents the total amount of thrombin generated), peak (the maximum concentration of thrombin produced), and velocity index (the slope between the start of thrombin formation and the peak). To rule out any "contamination" of unfractioned heparin (UFH), anti-Xa levels were determined in all samples. Only samples with undetectable anti-Xa $(<0.05 \mathrm{U} \mathrm{ml})$ were considered to be free of UFH and only these samples were included in the analysis.

To assess platelet reactivity, platelet-rich plasma, obtained by centrifuging whole blood for $10 \mathrm{~min}$ at $200 \mathrm{~g}$, was stimulated with $10 \mu \mathrm{M}$ adenosine diphosphate (ADP; Mascia Brunelli, Milan, Italy), and with $1 \mathrm{mM}$ arachidonic acid (AA; Sigma-Aldrich, Milan, Italy) and aggregation was assessed using a APACT 4 light transmission aggregometer (Helena Laboratories, Milan, Italy) as previously reported [18]. We defined patients with high platelet reactivity (HPR) those with platelet aggregation by $A A \geq 20 \%$ and/or by ADP $\geq 70 \%$ [19-22].

All data were prospectively collected and entered into a central database. Clinical follow-up information was obtained by contacting all the patients. All possible information derived also from hospital readmission or by referring physician, relatives, or municipality live registries were entered into the prospective database. All deaths were considered cardiac unless an unequivocal non cardiac cause could be documented.

Statistical analysis was performed using the SPSS (Statistical Package for Social Sciences, Chicago, IL, USA) software for Windows (Version 21.0). Values are presented as median and interquartile range (IQR). The Mann-Whitney test for unpaired data was used for comparison between two groups. Dichotomous variables were compared by $\mathrm{Chi}^{2}$ test. A receiver operating characteristic curve (ROC) analysis was used to determine the ability of TG parameters in predicting cardiovascular death at 24 months. The optimal cut-off point was calculated by determining the TG parameters that provided the greatest sum of sensitivity and specificity. We defined patients with residual TG those with ETP $\geq$ $1027.2 \mathrm{nM} / \mathrm{min}$ and/or peak $\geq 139.28 \mathrm{nM}$ and/or velocity index $\geq 35.7$ $\mathrm{nmol} / \mathrm{min}$. Cumulative survival curves were constructed by the Kaplan Meier method, and the log-rank test was used to assess statistical differences between survival curves. In order to test the independent association between TG parameters and cardiovascular death, we performed a multiple logistic regression analysis adjusted for an established risk score which has been extensively validated such as the Global Registry of Acute Coronary Events (GRACE) risk score (which includes age, heart rate, systolic blood pressure, creatinine, Killip class, elevated cardiac enzymes, ST-segment deviation, cardiac arrest at admission). All odds ratios (OR) are given with their 95\% confidence interval (CI). $\mathrm{p}<0.05$ was considered to be statistically significant.

\section{Results}

We investigated thrombin generation in 292 ( 217 men/75 women) ACS patients (155 ST-elevation myocardial infarction [STEMI]; 137 NSTEMI/Unstable Angina).

TG parameters did not significantly differ in relation to age, sex, smoking habit, hypertension, dyslipidemia, diabetes and leukocyte number (data not shown). Higher values of peak (192.21; IQR 141.73249.35) and velocity index (59.58; IQR 42.22-100.89) were found in ACS patients with renal failure respect to those without renal failure [peak 119.33 (IQR 61.21-200.42); velocity index 30.62 (IQR 12.62- 
Table 1

Clinical characteristics of patients investigated.

\begin{tabular}{|c|c|c|c|c|}
\hline & $\begin{array}{l}\text { Overall group } \\
\mathrm{n}=294(\%)\end{array}$ & $\begin{array}{l}\text { CV death } \\
n=57(\%)\end{array}$ & $\begin{array}{l}\text { No CV death } \\
\mathrm{n}=237(\%)\end{array}$ & $\mathrm{p}^{\mathrm{b}}$ \\
\hline Age, years ${ }^{c}$ & $76(65-84)$ & $86(80-90)$ & $72(62-81)$ & 0.000 \\
\hline Male gender, n (\%) & $218(74.1)$ & $33(57.9)$ & $185(78.1)$ & 0.0037 \\
\hline Diabetes, n (\%) & $80(27.2)$ & $20(35.1)$ & $60(25.3)$ & NS \\
\hline Smoking habit, n (\%) & $181(61.6)$ & $29(50.1)$ & $152(64.1)$ & 0.0703 \\
\hline Hypertension, n (\%) & 185 (62.9) & $38(66.7)$ & $147(62.0)$ & NS \\
\hline Dyslipidemia, n (\%) & $119(40.5)$ & $21(36.8)$ & $98(41.4)$ & NS \\
\hline Renal failure ${ }^{\mathrm{a}}, \mathrm{n}(\%)$ & $14(4.8)$ & $8(14.0)$ & $6(2.5)$ & 0.0015 \\
\hline STEMI, n (\%) & $157(53.4)$ & $24(42.1)$ & $133(56.1)$ & NS \\
\hline ACE-inhibitors, n (\%) & $70(23.8)$ & $21(36.8)$ & $49(20.1)$ & NS \\
\hline Ca-antagonists, n (\%) & $62(21.1)$ & $16(28.1)$ & $46(19.4)$ & NS \\
\hline Beta-blockers, n (\%) & $70(23.8)$ & $21(36.8)$ & $49(20.1)$ & 0.0147 \\
\hline Antiarrhythmic, n (\%) & $17(5.8)$ & $4(7.0)$ & $13(5.5)$ & NS \\
\hline Statins, n (\%) & $76(25.9)$ & $16(28.1)$ & $60(25.3)$ & NS \\
\hline Nitrates, n (\%) & $44(15.0)$ & $14(24.6)$ & $30(12.7)$ & 0.0366 \\
\hline AntillbIIIa & $6(2.0)$ & $1(1.8)$ & $5(2.1)$ & NS \\
\hline Killip class 1 & $195(66.3)$ & $33(57.9)$ & $162(68.4)$ & 0.000 \\
\hline Killip class $>1$ & $53(18.0)$ & $24(42.1)$ & $21(8.9)$ & \\
\hline One vessel treated, n (\%) & $94(32.0)$ & $15(26.3)$ & $79(33.3)$ & NS \\
\hline Two vessel treated, n (\%) & $103(35.0)$ & $20(35.1)$ & $83(35.0)$ & \\
\hline Three vessel treated, n (\%) & $97(33.0)$ & $22(38.6)$ & $75(31.6)$ & \\
\hline
\end{tabular}

$\mathrm{CV}$ : cardiovascular death.

a Renal insufficiency defined by creatinine levels above $2.0 \mathrm{mg} / \mathrm{dl}$.

b CV death vs no CV death.

c Values are expressed as median and (range).

66.40)] ( $\mathrm{p}<0.005$ ). No significant differences in TG values according to Killip class and STEMI /NSTEMI were found (data not shown).

At two-year follow-up, 57 out of 292 patients (19.5\%) died from cardiovascular causes. Cardiac mortality rate was $11.2 \%(\mathrm{n}=33)$ at 6 months and 9.2\% $(\mathrm{n}=24)$ at $6-24$ months. With regards to cardiovascular risk factors, patients who died during the follow-up were significantly older, with a higher prevalence of females and renal failure, and with a higher Killip class as compared to patients who remained alive (Table 1).

Among hematological parameters, patients who died during followup showed significant lower values of hemoglobin, hematocrit, red blood cells and a significantly higher number of leukocytes (Table 2).

Higher values of ETP, peak and velocity index were detected in dead compared to alive patients (Table 2 ).

The landmark analysis using the prespecified starting point of 6 months showed that the differences between groups according to cardiac mortality emerged in the short-term follow-up as well as from 6 months to long term.

Receiver operating characteristics (ROC) curve analyses (Fig. 1) identified ETP $=1027.2 \mathrm{nMxmin}$, peak $=139.28 \mathrm{nM}$, and velocity index $=35.7 \mathrm{nmol} / \mathrm{min}$ as the optimal cut-off values to predict cardiovascular death [ETP area under curve 58\% (50-67), p = 0.029; peak area under the curve $63 \%$ (54-72), $\mathrm{p}=0.002$; velocity index area under the curve 65\% (56-74), $\mathrm{p}<0.001$ ].

By using these cut-off values, significantly higher percentages of patients with ETP $\geq 1027.2 \mathrm{nM} / \mathrm{min}$, peak $\geq 139.28 \mathrm{nM}$, and velocity index $\geq 35.7 \mathrm{nmol} / \mathrm{min}$ were observed among those who died compared to those patients who remained alive [ETP: 33 (57.9\%) vs 100 (42.2\%), $\mathrm{p}=0.039$; peak: 35 (61.4\%) vs 92 (38.8\%), $\mathrm{p}=0.003$; velocity index: 36 (63.2\%) vs 90 (37.9\%), $\mathrm{p}=0.001]$.

Kaplan Meier curves for cardiovascular death according to higher ETP, peak, and velocity index significantly diverged (Fig. 2).

In order to evaluate the association of thrombin generation with the occurrence of cardiovascular death we performed a logistic regression analysis. In the univariate model ETP, peak, and velocity index, together with age, female sex, leukocyte number, renal failure and Killip class, were significantly associated with the risk of cardiovascular death (Table 3). At the multivariate analysis, adjusted for the traditional cardiovascular risk factors (age, male gender, smoking, hypertension, dyslipidemia, diabetes mellitus) and for the Global Registry of Acute Coronary Events (GRACE) risk score variables, ETP, peak, velocity index, leukocyte number, and age remained significantly associated with the risk of cardiovascular death (Table 3 ).

In our population, the prevalence of HPR by ADP and/or by AA was $33.9 \%(\mathrm{n}=99)$. Cardiovascular mortality rate was $29.3 \%(29 / 99)$ in the HPR by ADP and/or by AA and 14.5\% (28/193) in the low residual platelet reactivity group $(\mathrm{p}=0.004)$. At univariate logistic regression analysis high residual platelet reactivity was a significant predictor of mortality [OR: 2.31 (95\% CI 1.28-4.18), $\mathrm{p}=0.006$ ]. After adjustment for the traditional cardiovascular risk factors and for the Global Registry of Acute Coronary Events (GRACE) risk score variables, HPR remained independently associated with mortality [OR 2.09; 95\% CI 1.07-4.07), $\mathrm{p}=0.031]$.

At a multivariate logistic regression analysis in a full model including HPR, thrombin generation variables and the other independent variables, HPR, ETP $\geq 1027.2 \mathrm{nM} / \mathrm{min}$, peak $\geq 139.28 \mathrm{nM}$, and velocity index $\geq 35.7 \mathrm{nmol} / \mathrm{min}$, remained significantly and independently associated with cardiovascular mortality (HPR: OR 2.51 (95\% CI 1.17-5.40), $\mathrm{p}=0.018$; ETP: OR 2.35 (95\% CI 1.11-5.00), $\mathrm{p}=0.027$; peak: OR 2.42 (95\% CI 1.13-5.15), p = 0.022; velocity index: OR 2.43 (95\% CI 1.145.20), $\mathrm{p}=0.022]$.

\section{Discussion}

In the present study we have found that: 1 ) the entity of thrombin generation after PCI with stent implantation is an independent predictor of cardiovascular death in ACS patients; 2 ) the risk of cardiovascular death associated with thrombin generation is independent of platelet reactivity.

Table 2

Laboratory parameters of patients investigated.

\begin{tabular}{|c|c|c|c|c|}
\hline & $\begin{array}{l}\text { Overall group } \\
(\mathrm{n}=294)\end{array}$ & $\begin{array}{l}\text { CV death } \\
(\mathrm{n}=57)\end{array}$ & $\begin{array}{l}\text { No CV death } \\
(\mathrm{n}=237)\end{array}$ & $\mathrm{p}^{\mathrm{a}}$ \\
\hline ETP (nM/min) & $986(665-1269)$ & $1116(701-1441)$ & $940(664-1260)$ & 0.048 \\
\hline Peak (nM) & $117(61-193)$ & $176(81-259)$ & $107(60-181)$ & 0.002 \\
\hline Velocity index (nM/min) & $30(13-63)$ & $62(21-98)$ & $26(12-50)$ & 0.000 \\
\hline Leukocytes $\left(\times 10^{3} / \mu \mathrm{l}\right)$ & $9645(7325-12,575)$ & $11,350(8608-14,675)$ & $9220(6975-12,100)$ & 0.004 \\
\hline Red blood cells $\left(\times 10^{6} / \mu \mathrm{l}\right)$ & $4.64(4.24-4.99)$ & $4.39(3.72-4.76)$ & $4.72(4.39-5.03)$ & 0.001 \\
\hline Hemoglobin (g/dl) & $14.0(12.5-15.0)$ & $12.7(11.2-13.9)$ & $14.1(13.0-15.2)$ & 0.000 \\
\hline Hematocrit (\%) & $41.4(36.9-44.5)$ & $37.6(33.4-41.8)$ & $42.3(38.7-44.9)$ & 0.000 \\
\hline Platelets $\left(\times 10^{3} / \mu \mathrm{l}\right)$ & $225(191-268)$ & $233(197-264)$ & $222(189-270)$ & NS \\
\hline Creatinine (mg/dl) & $0.93(0.76-1.11)$ & $1.10(0.86-1.70)$ & $0.90(0.74-1.06)$ & 0.000 \\
\hline CK-MB (ng/ml) & $67.7(18.8-176.8)$ & $43.00(19.6-185.6)$ & $75.95(15.5-175.4)$ & NS \\
\hline AA and/or ADP-HRPR, n (\%) & 99 (33.7) & $29(50.9)$ & $70(29.5)$ & 0.002 \\
\hline
\end{tabular}

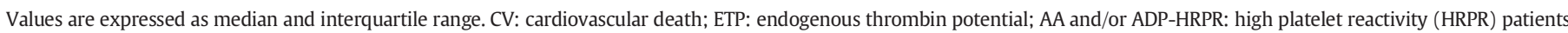
with platelet aggregation by $\mathrm{AA} \geq 20 \%$ and/or by $\mathrm{ADP} \geq 70 \%$; $\mathrm{CK}-\mathrm{MB}$ : Creatine Kinase $\mathrm{MB}$.

a CV death vs no CV death. 
1) ETP

ROC Plot

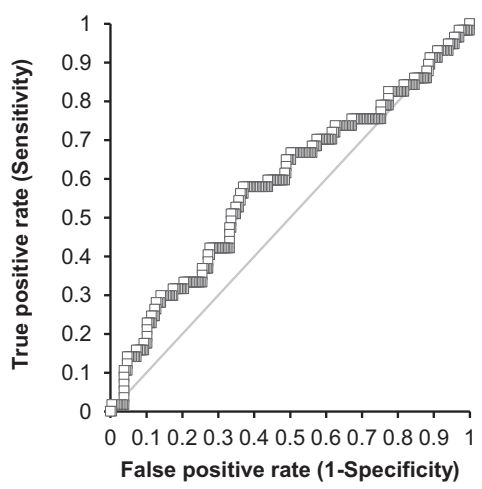

2) PEAK

ROC Plot

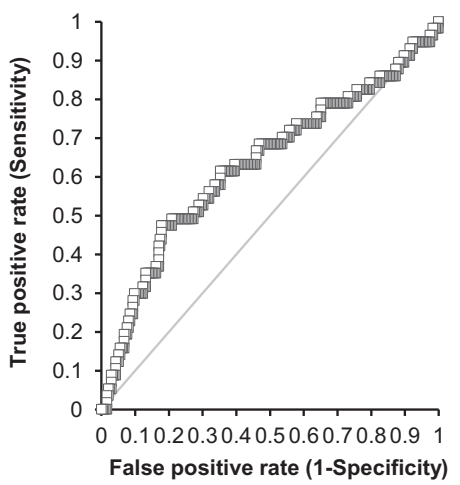

3) VELOCITY INDEX

ROC Plot

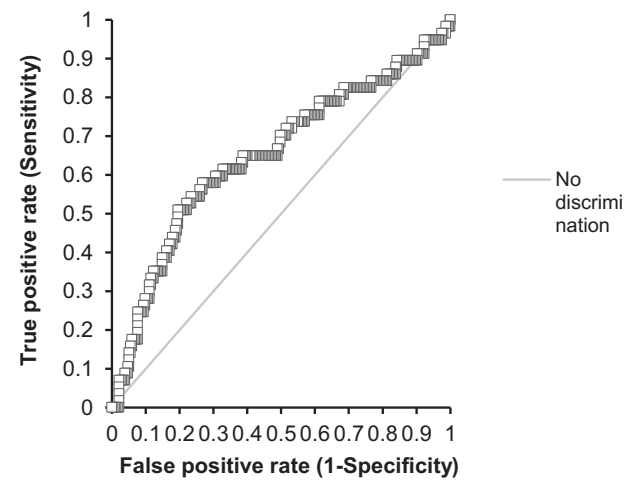

Fig. 1. Receiver-operating characteristic curves for: endogenous thrombin potential (ETP) (1), peak (2) and velocity index (3).

In our study, the clinical variables associated with an increased risk of cardiovascular death at multivariate analysis were advanced age, and Killip class $>1$. As regards laboratory parameters, white blood cell count (>10,000 cells/ $\mu \mathrm{l}$ ) was significantly higher in patients with unfavorable prognosis and independent predictor of death at multivariate analysis. This confirms that the exaggerate inflammatory response occurring in these patients contributes to the worse prognosis $[23,24]$.

As regards thrombin generation, ETP, peak thrombin and velocity index were significantly higher in patients who died compared to alive patients during 24-month follow-up. At multivariate analysis ETP, peak thrombin and velocity index remained significantly associated with cardiovascular death.
It is well known that blood clotting activation, mostly evaluating single markers such as fibrinopeptide A, prothrombin fragment $1+2$ and D-dimer, is present in ACS patients and it is associated with unfavorable outcomes $[25,4,26]$. The majority of these evidences derived from studies conducted when invasive procedures and stent implantation were less extensively performed. In the era of $\mathrm{PCI}$ and stenting with dual antiplatelet therapy, two papers evaluated thrombin generation in CAD patients. In 2011 Smid and coworkers [9] in a small group of AMI patients obtained no conclusive results of the role of hypercoagulability in determining the risk of recurrent ischemic cardiovascular complications due to the low percentage of clinical events. In 2014 the LURIC study observed in a large, but non homogeneous cohort of CAD patients

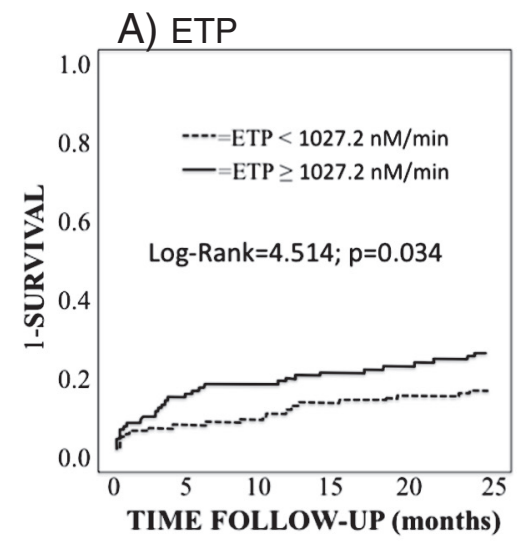

B) Peak

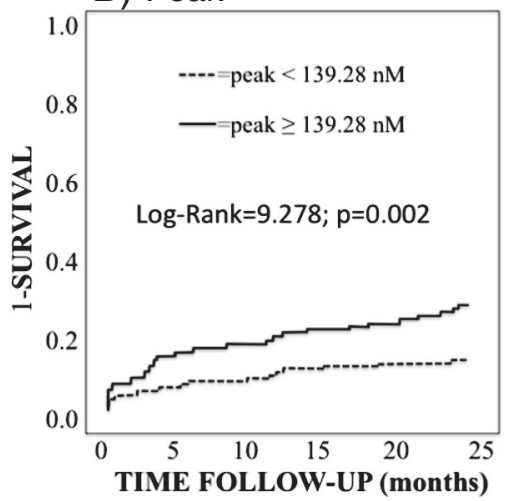

C) Velocity index

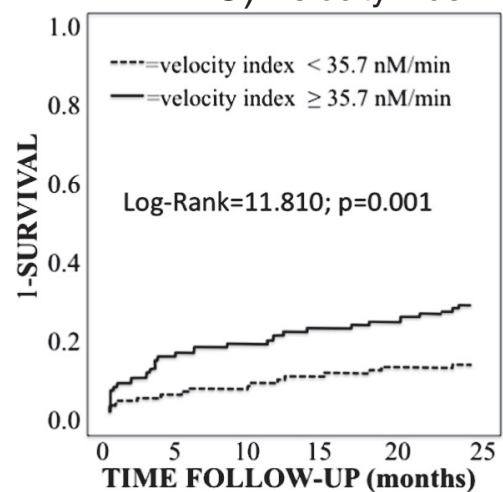

Fig. 2. Kaplan-Meier curves for cardiovascular death according to elevated values of endogenous thrombin potential (ETP) (A), peak (B) and velocity index (C). 
Table 3

Predictors of cardiovascular death.

\begin{tabular}{|c|c|c|c|c|c|c|}
\hline & \multicolumn{3}{|c|}{ Univariate analyses } & \multicolumn{3}{|c|}{ Multivariate analyses } \\
\hline & OR & $(95 \% \mathrm{CI})$ & $\mathrm{p}$ & OR & $(95 \% \mathrm{CI})$ & $\mathrm{p}$ \\
\hline $\mathrm{ETP} \geq 1027.2 \mathrm{nMxmin}$ & 1.86 & $1.03-3.34$ & 0.039 & 2.58 & $1.10-6.03$ & 0.029 \\
\hline Peak $\geq 139.28$ nM & 2.47 & $1.37-4.48$ & 0.003 & 3.27 & $1.35-7.92$ & 0.009 \\
\hline $\begin{array}{l}\text { Velocity index } \geq 35.7 \\
\mathrm{nM} / \mathrm{min}\end{array}$ & 2.76 & $1.52-5.03$ & 0.001 & 3.06 & $1.27-7.39$ & 0.013 \\
\hline Age (years) & 1.15 & $1.10-1.20$ & 0.000 & 1.17 & $1.10-1.23$ & $<0.001$ \\
\hline Sex, female & 2.62 & $1.43-4.83$ & 0.002 & 0.92 & $0.39-2.20$ & 0.857 \\
\hline Renal failure & 7.63 & $2.21-26.40$ & 0.001 & 6.49 & $0.61-18.67$ & 0.120 \\
\hline Killip class & 1.45 & $1.10-1.92$ & 0.010 & 1.54 & $0.99-2.39$ & 0.055 \\
\hline Leukocyte number & 1.13 & $1.05-1.22$ & 0.002 & 1.21 & $0.9-1.35$ & $<0.001$ \\
\hline
\end{tabular}

CI: confidence interval; ETP: endogenous thrombin potential; OR: odd ratio.

(unstable and stable coronary disease) an inverse association between cardiovascular death and ETP, investigated within the first month from the index event [10].

Our findings may appear discordant from these results which underpin the thrombin-paradox. Nevertheless, clinical setting and timing of the evaluation were not comparable with our study. In our setting we explored thrombin generation in ACS patients within $24 \mathrm{~h}$ from clopidogrel loading and periprocedural heparin administration in patients undergoing PCI with stent implantation. Furthermore our patients were older, with higher prevalence of traditional cardiovascular risk factors and with a higher cardiovascular risk, as shown by the different incidence of mortality.

We found that, despite an optimal antithrombotic therapy and a periprocedural anticoagulant treatment, in a subset of patients (43\%) a residual thrombin generation is yet detectable after PCI. It is known that ACS is associated with a hypercoagulability state and for this reason it is essential to use anticoagulant treatment in the management of these patients. Nevertheless, we have demonstrated that, on the top of antithrombotic treatment performed according to guidelines, in some patients a residual thrombin generation is detectable after PCI. These patients have a significantly higher risk of long-term cardiovascular death.

On the other hand, a large body of evidence has documented the association between the entity of platelet reactivity on treatment and the risk of cardiac death, stent thrombosis and ischemic recurrences. In this study we have confirmed this datum. Additionally, our results suggest that both residual thrombin generation and platelet reactivity are associated - but independently - with cardiovascular death. From a clinical point of view, these results are supporting the presence of patients in which it might be more relevant to obtain an adequate platelet or clotting inhibition.

Thrombin is one of the more potent platelet activators and hyper-reactive platelets affect clotting system. Results obtained at multivariate analysis indicate that thrombin generation and platelet reactivity are two distinct phenomena affecting independently cardiovascular outcome. However, in this study patients were on dual antiplatelet therapy with aspirin and clopidogrel; so, we cannot exclude variations on these results in the presence of the new and more potent antiplatelet drugs.

Limitations of our study are that we measured TG at one time only, that we observed as endpoint the cardiovascular death only, and that we did not measure markers of thrombin generation, nor other proteins, known to be positive determinants of thrombin generation. Another limitation of the study is that we did not analyzed thrombophilic polymorphisms.

Our study adds new evidence that thrombin generation parameters could be useful biomarkers for ACS risk stratification immediately after PCI. The measurement of thrombin generation might help clinicians to detect ACS patients at higher risk of adverse event during the followup, who could obtain advantage by a prolonged antithrombotic treatment.

\section{Disclosures}

Dr. Marcucci has received honoraria for lectures from DaiichiSankyo/Eli Lilly and Merck Sharp Dohme. Dr. Antoniucci has received consulting fees from Daiichi-Sankyo/Eli Lilly and The Medicines Company. Dr. Abbate has received consulting fees from Eli Lilly; lecture fees from Instrumentation Laboratory and Sigma Tau; and research grant funding from Bayer, Boehringer Ingelheim, and Pfizer. Dr. Gensini has received support from Bayer, Boehringer Ingelheim, Eli Lilly; lecture fees from AstraZeneca, GlaxoSmithKline, Instrumentation Laboratory, Menarini, and Sigma Tau; research grant funding from Novo Nordisk, Merck Sharp Dohme, Pfizer, Pierrel, Sanofi, and Servier; and honorarium from Scharper. All other authors have no relationships relevant to the contents of this paper to disclose.

\section{Acknowledgements}

This study was supported by grants from Italian Ministry of Health (11RFRM and 12RFRM), 2011 and 2012 Finalized Research Programs.

\section{References}

[1] G.G. Neri Serneri, G.F. Gensini, M. Carnovali, et al., Association between time of increased fibrinopeptide A levels in plasma and episodes of spontaneous angina: a controlled prospective study, Am. Heart J. 113 (3) (1987) 672-678.

[2] Y. Lopez, M.J. Paloma, J. Rifon, B. Cuesta, J.A. Páramo, Measurement of prethrombotic markers in the assessment of acquired hypercoagulable states, Thromb. Res. 93 (1999) 71-78.

[3] G.D.O. Lowe, Circulating inflammatory markers and risks of cardiovascular and noncardiovascular disease, J. Thromb. Haemost. 3 (2005) 1618-1627.

[4] D. Ardissino, P.A. Merlini, K.A. Bauer, et al., Coagulation activation and long-term outcome in acute coronary syndromes, Blood 102 (2003) 2731-2735.

[5] V.G. Kalaria, W. Zoreba, A.J. Moss, et al., Gender-related differences in thrombogenic factors predicting recurrent cardiac events in patients after acute myocardial infarction, Am. J. Cardiol. 85 (2000) 1401-1408.

[6] S.G. Thompson, J. Kienast, S.D.M. Pyke, F. Haverkate, J.C.W. van de Loo, Haemostatic factors and the risk of myocardial infarction or sudden death in patients with angina pectoris. European Concerted Action on Thrombosis and Disabilities Angina Pectoris Study Group, N. Engl. J. Med. 332 (1995) 635-641.

[7] F. Sofi, R. Marcucci, A.M. Gori, R. Abbate, G.F. Gensini, Residual platelet reactivity on aspirin therapy and recurrent cardiovascular events-a meta-analysis, Int. J. Cardiol. 128 (2) (2008 Aug 18) 166-171.

[8] S.S. Brar, J. ten Berg, R. Marcucci, et al., Impact of platelet reactivity on clinical outcomes after percutaneous coronary intervention. A collaborative metaanalysis of individual participant data, J. Am. Coll. Cardiol. 58 (19) (2011 Nov 1) 1945-1954.

[9] M. Smid, A.W. Dielis, M. Winkens, et al., Thrombin generation in patients with a first acute myocardial infarction, J. Thromb. Haemost. 9 (3) (2011) 450-456.

[10] J.G. Schneider, B. Isermann, M.E. Kleber, et al., Inverse association of the endogenous thrombin potential (ETP) with cardiovascular death: the Ludwigshafen Risk and Cardiovascular Health (LURIC) study, Int. J. Cardiol. 176 (1) (2014) 139-144.

[11] H.C. Hemker, P. Giesen, R. Aldieri, et al., The calibrated automated thrombogram (CAT): a universal routine test for hyper- and hypocoagulability, Pathophysiol. Haemost. Thromb. 32 (2002) 249-253.

[12] F. Cesari, R. Marcucci, A.M. Gori, et al., Reticulated platelets predict cardiovascular death in acute coronary syndrome patients, Thromb. Haemost. 109 (2013) 846-853.

[13] C.W. Hamm, J.P. Bassand, S. Agewall, et al., ESC guidelines for the management of acute coronary syndromes in patients presenting without persistent ST-segment elevation, Eur. Heart J. 32 (2011) 2999-3054.

[14] R. Cifkova, S. Erdine, R. Fagard, et al., Practice guidelines for primary care physicians: 2003 ESH/ESC hypertension guidelines, J. Hypertens. 21 (2003) 1779-1786

[15] Report of the expert committee on the diagnosis and classification of diabetes mellitus, Diabetes Care 26 (2003) 5-20.

[16] Third report of the National Cholesterol Education Program (NCEP) expert panel on detection, evaluation, and treatment of high blood cholesterol in adult (Adult Treatment Panel III) final report, Circulation 106 (2002) 3143-3421.

[17] H.C. Hemker, P. Giesen, R. Al Dieri, et al., Calibrated automated thrombin generation measurement in clotting plasma, Pathophysiol. Haemost. Thromb. 33 (2003) 4-15

[18] R. Paniccia, E. Antonucci, A.M. Gori, et al., Different methodologies for evaluating the effect of clopidogrel on platelet function in high-risk coronary artery disease patients, J. Thromb. Haemost. 5 (2007) 1839-1847.

[19] P.A. Gum, K. Kottke-Marchant, P.A. Welsh, J. White, E.J. Topol, A prospective blinded determination of the natural history of aspirin resistance among stable patients with cardiovascular disease, J. Am. Coll. Cardiol. 41 (2003) 961-965. 
[20] T. Geisler, H. Langer, M. Wydymus, et al., Low response to clopidogrel is associated with cardiovascular outcome after coronary stent implantation, Eur. Heart J. 27 (2006) 2420-2425.

[21] P. Buonamici, R. Marcucci, A. Migliorini, et al., Impact of platelet reactivity after clopidogrel administration on drug-eluting stent thrombosis, J. Am. Coll. Cardiol. 49 (2007) 2312-2317.

[22] R. Paniccia, E. Antonucci, A.M. Gori, et al., Comparison of different methods to evaluate the effect of aspirin on platelet function in high-risk patients with ischemic heart disease receiving dual antiplatelet treatment, Am. J. Clin. Pathol. 128 (2007) 143-149.

23] D.W. Park, S.W. Lee, S.C. Yun, et al., A point-of-care platelet function assay and C-reactive protein for prediction of major cardiovascular events after drug-eluting stent implantation, J. Am. Coll. Cardiol. 58 (25) (2011) 2630-2639.
[24] L.P. He, X.Y. Tang, W.H. Ling, W.Q. Chen, Y.M. Chen, Early C-reactive protein in the prediction of long-term outcomes after acute coronary syndromes: a meta-analysis of longitudinal studies, Heart 96 (5) (2010) 339-346.

[25] G.G. Serneri, R. Abbate, A.M. Gori, et al., Transient intermittent lymphocyte activation is responsible for the instability of angina, Circulation 86 (3) (1992) 790-797.

[26] P.E. Morange, C. Bickel, V. Nicaud, et al., Haemostatic factors and the risk of cardiovascular death in patients with coronary artery disease: the AtheroGene study, Arterioscler. Thromb. Vasc. Biol. 26 (12) (2006) 2793-2799. 